\title{
Does captivity alter problem-solving behavior in Goffin's cockatoos?
}

\author{
Irene M. Pepperberg ${ }^{1}$
}

Accepted: 11 December 2020 / Published online: 24 February 2021

(C) The Psychonomic Society, Inc. 2020

\begin{abstract}
Summary
Researchers often argue for the existence of "captivity bias": a higher success rate that may be observed for nonhumans tested in captivity compared to those tested in nature, purportedly because the former have certain advantages that the latter lack. Rössler et al. (Scientific Reports 10, 8681, 2020) find that, at least for Goffin's cockatoos (Cacatua goffiniana), differences exist in motivation, not in problem-solving ability.
\end{abstract}

Keywords Goffin's cockatoo $\cdot$ Captivity bias $\cdot$ Innovation $\cdot$ Parrot cognition $\cdot$ Parrot motivation

"Captivity bias" is a critical issue when studying nonhuman cognition - specifically, what effects do living conditions and life history have on nonhumans' success in solving cognitive problems? Often, long-term captive subjects outperform their wild-living counterparts (e.g., hyenas, Crocuta crocuta, Benson-Amram, Weldele, \& Holekamp, 2013; keas, Nestor notabilis, Gajdon, Fijn, \& Huber, 2004). Several reasons for such differences have been proposed, the most obvious being enculturation - i.e., familiarity with human activities on which the tasks being tested are based and/or previous experience with somewhat familiar tasks. Researchers also argue that captive subjects can perhaps re-direct resources (energetic or cognitive) no longer required for foraging or avoiding predation to perform the task (reviewed in Benson-Amram et al., 2013), or that in the field dominant subjects may prevent subordinates from accessing the apparatus for the amount of time necessary for devising a solution (Gajdon et al., 2004). Rössler et al. (2020) argue instead that the observed behavioral differences, at least for Goffin's cockatoos (Cacatua goffiniana), are only in motivation - that captive subjects may, for example, simply interact more readily with the apparatus for reasons related to boredom, play, or from expectancies of rewards based on prior experience (see also Smith, Greene, Hartsfield, \& Pepperberg, 2021). Rössler et al. (2020) show compelling evidence that for their subjects that

Irene M. Pepperberg

impepper@media.mit.edu

1 Department of Psychology, Harvard University, Cambridge, MA, USA do engage in the tasks, levels of successful outcomes differ little between laboratory and wild-caught individuals.

Rössler et al.'s (2020) investigation is notable in its striking methodological difference from previous studies that examine captivity bias. Such studies, which they review, usually engage subjects in a single task or at most a small set of assays; data from subjects challenged by only a limited range of tasks are particularly susceptible to being influenced by issues such as enculturation or pre-exposure. Rössler et al. (2020), however, presented their parrots with a large battery of tasks (i.e., 20), allowing subjects to tackle them in their own preferred order. The assays, contained in plexiglass boxes, were arranged in a semi-circle arena and birds were given individual access; thus, no single subject could monopolize the apparatus. Tasks were designed to require a variety of actions and test the emergence of innovative behavior patterns. By solving each problem, birds could acquire a food reward - slivers of cashew in the laboratory and corn kernels in the wild. Some tasks appeared difficult for both wild-caught and laboratory birds (e.g., unbending a wire in a particular direction to release a trap door); others (e.g., pulling on a drawer handle to provide access) seem to have been quickly solved by both sets of subjects. On only a very few tasks (e.g., guiding a reward off a shelf, pressing a button attached to a dowel that then pushed a reward off a shelf); did laboratory birds show a decided advantage over the wild-caught subjects (however, possible experience with button-pressing was noted); in only a few others (e.g., rotating a wheel, squeezing a clip) did wild birds significantly outperform their captive brethren. Thus, overall, few differences emerged in demonstrated abilities, and predispositions toward capacities required for particular 
tasks were likely balanced. However, participation rates were $<40 \%$ for wild-caught birds versus $\sim 90 \%$ for laboratory birds.

I do not discount Rössler et al.'s (2020) explanation that their observed differences were primarily motivational, but wonder whether some underlying, if related, factors may have also been responsible for their results. Specifically, might short-term captivity affect behavior and behavioral predispositions? Did task similarity swamp possible innovative disparity? I discuss each possibility in turn.

That individual differences exist across nonhumans is no longer disputed; might certain personality factors relating to motivation be fostered in nature that are less useful in captivity? For example, captive Amazona amazonica subjects differ in degrees of attentional bias, which occurs "...either because individuals attend more quickly to certain environmental stimuli, or because they cannot disengage their attention from such stimuli" (Cussen \& Mench, 2014, p. 123). In nature, threat of predation favors birds with a predilection for high attentional bias, but those captive parrots that exhibited high attentional bias were less adept at the cognitive tasks that were administered, mainly because they balked when engaging with the apparatus (Cussen \& Mench, 2014). Although one might argue that both wild-caught and captive cockatoos would have demonstrated a similar range of personality differences when compared to the Amazon parrots, possibly any existent predisposition toward high attentional bias would be bolstered in wild cockatoos that had recently been trapped by humans, and thus might affect their reticence to engage with the apparatus, even if they were well habituated.

Might the specific circumstances of short-term captivity have affected some behavioral patterns? Although the wildcaught birds were likely from the same flock given where they were trapped, might removal to the captive aviary have disrupted a stable dominance hierarchy, such that the subjects were now exerting considerable effort in establishing a new (literal) pecking order? Such concerns may also have affected their attentional bias and willingness to engage in activities in the foraging arena - for example, might jockeying for position have sapped cognitive and energetic resources, or might they have viewed removal from the aviary, even for short periods, as being detrimental to their newly acquired position and thus be responsible for their reluctance to engage in the tasks in the arena? In contrast, might it also be possible that recently wildcaught individuals that normally spend considerable time and effort on foraging and predation avoidance, but that are now fed pretty much ad libitum, protected, and without enough time in captivity to engender boredom, have simply been content for the short-term to freeload, particularly as the corn rewards were part of their daily diet?

My final comment involves how the tasks themselves might have engendered similar results among motivated subjects in both groups; that is, might similarities in the solutions to a subset of tasks have swamped other effects? From what I could see in the videos and figures, although a number of tasks at first glance appeared to differ in their patterns of action, on further investigation the range of motions seemed somewhat constrained. Several tasks (seesaw, swish, shovel, plank, flip-box, shelf) all required the subject to grasp an external piece of plastic that was embedded in a box; once the plastic was grasped, however, the ways in which the plastic could actually be manipulated were limited. Cockatoos are notoriously capable of using their innovative abilities to game a system, finding simpler - albeit often extremely clever! - solutions to various problems; here it appeared that all birds figured out fairly quickly that after grabbing the plastic only a few available options existed; they then either used only small adaptations or learned that the physical constraints meant that any allowed motion would solve other, similar tasks. Thus, any real differences in wild-caught versus laboratory birds' success might have been overshadowed by the simplicity of the transfer. It would be fascinating to see how both sets of birds would respond to tasks that allowed considerably greater ranges of motion how quickly might these intelligent birds settle on the correct solutions?

In sum, whether or not the factors I suggest influenced the behavioral patterns observed by Rössler et al. (2020), and even if their cockatoos were (cleverly) gaming the system, their study provides an important addition to the field. They examine critical issues not fully addressed in previous studies. Only by continuing to refine the types of experiments we administer, and only by carefully adapting our tasks so as not to preferentially benefit either wild-caught or laboratory subjects, will we be able to uncover the true capacities of nonhumans.

Data availability The article contains no data.

\section{References}

Benson-Amram, S., Weldele, M.L., \& Holekamp, K.E. (2013). A comparison of innovative problem-solving abilities between wild and captive spotted hyaenas, Crocuta crocuta. Animal Behaviour, 85, 349-356.

Cussen, V.A., \& Mench, J.A. (2014). Personality predicts cognitive bias in captive psittacines, Amazona amazonica. Animal Behaviour, 89, 123-130.

Gajdon, G.K., Fijn, N., \& Huber, L. (2004). Testing social learning in a wild mountain parrot, the kea (Nestor notabilis). Animal Learning \& Behavior, 32, 62-71.

Rössler, T., Mioduszewka, B., O’Hara, M., Huber, L., Prawiradilaga, D.M., \& Ausperg, A.M.I. (2020). Using an Innovation Arena to compare wild-caught and laboratory Goffin's cockatoos. Scientific Reports, 10, 8681 .

Smith, G.E., Greene, D.E., Hartsfield, L.A., \& Pepperberg, I.M. (2021). The play's the thing: Initial evidence for eliciting contrafreeloading in Grey parrots (Psittacus erithacus) via the opportunity for playful foraging. Manuscript submitted for publication.

Publisher's note Springer Nature remains neutral with regard to jurisdictional claims in published maps and institutional affiliations. 